\title{
BMJ Open Protocol for a scoping review study to identify and classify patient-centred quality indicators
}

\author{
Rachel J Jolley, ${ }^{1}$ Diane L Lorenzetti, ${ }^{1,2}$ Kimberly Manalili, ${ }^{1}$ Mingshan Lu, ${ }^{1,3}$ \\ Hude Quan, ${ }^{1}$ Maria J Santana ${ }^{1}$
}

To cite: Jolley RJ,

Lorenzetti DL, Manalili K, et al. Protocol for a scoping review study to identify and classify patient-centred quality indicators. BMJ Open 2016;6:e013632. doi:10.1136/bmjopen-2016013632

- Prepublication history and additional material is available. To view please visit the journal (http://dx.doi.org/ 10.1136/bmjopen-2016013632)

Received 27 July 2016 Revised 12 October 2016 Accepted 20 October 2016

CrossMark

\footnotetext{
${ }^{1}$ Department of Community Health Sciences, University of Calgary, Calgary, Alberta, Canada

${ }^{2}$ Institute of Health Economics, Edmonton, Alberta, Canada

${ }^{3}$ Department of Economics, University of Calgary, Calgary, Alberta, Canada
}

Correspondence to Dr Maria J Santana; mjsantan@ucalgary.ca

\section{ABSTRACT}

Introduction: The concept of patient-centred care (PCC) is changing the way healthcare is understood, accepted and delivered. The Institute of Medicine has defined PCC as 1 of its 6 aims to improve healthcare quality. However, in Canada, there are currently no nationwide standards in place for measuring and evaluating healthcare from a patient-centred approach. In this paper, we outline our scoping review protocol to systematically review published and unpublished literature specific to patient-centred quality indicators that have been implemented and evaluated across various care settings.

Methods and analysis: Arksey and O'Malley's scoping review methodology framework will guide the conduct of this scoping review. We will search electronic databases (MEDLINE, EMBASE, the Cochrane Library, Cumulative Index to Nursing and Allied Health Literature (CINAHL), PsycINFO, Social Work Abstracts, Social Services Abstracts), grey literature sources and the reference lists of key studies to identify studies appropriate for inclusion. 2 reviewers will independently screen all abstracts and full-text studies for inclusion. We will include any study which focuses on quality indicators in the context of PCC. All bibliographic data, study characteristics and indicators will be collected and analysed using a tool developed through an iterative process by the research team. Indicators will be classified according to a predefined conceptual framework and categorised and described using qualitative content analysis.

Ethics and dissemination: The scoping review will synthesise patient-centred quality indicators and their characteristics as described in the literature. This review will be the first step to formally identify what quality indicators have been used to evaluate PCC across the healthcare continuum, and will be used to inform a stakeholder consensus process exploring the development of a generic set of patient-centred quality indicators applicable to multiple care settings. The results will be disseminated through a peer-reviewed publication, conference presentations and a one-day stakeholder meeting.

\section{BACKGROUND}

The concept of patient-centred care is changing the way healthcare is understood,

\section{Strengths and limitations of this study}

- This will be the first scoping review to identify quality indicators that are specific to patientcentred care across multiple different conditions and healthcare settings.

- The search strategy includes seven electronic databases with peer-reviewed literature, including article bibliographies and numerous conference proceedings, as well as a broad range of grey literature sources, including government and other organisation websites including quality improvement documents.

- Stakeholders including patient networks will be consulted and engaged throughout the study review process.

- Although this study will identify candidate indicators for further development, there will be no formal assessment of study quality or direct comparison of quality of the indicators.

accepted and delivered. Traditionally, the quality of healthcare has been driven by policymakers and evaluated through the lens of healthcare professionals and management only, often excluding the patient perspective. Stakeholders from across the continuum of care have called for evidence-informed system improvements to foster innovation and improve the delivery of care; recent policy emphasises that patient views complement healthcare provider perspectives, as well as provide unique information about healthcare effectiveness. ${ }^{1}$

In its landmark report, Crossing the Quality Chasm, the Institute of Medicine (IOM) outlined six aims for quality improvement, which include the core need for healthcare to be patient-centred. ${ }^{2}$ According to the IOM's definition, patient-centred care is care that aims to include the patients' values, needs and preferences in the healthcare system, ensuring that patient values guide all clinical decisions. ${ }^{2}$ This concept of patientcentred care has carried into other 
healthcare improvement strategies. The Institute for Healthcare Improvement has incorporated the aim of improving the patient experience of care as one of its three dimensions in its 'Triple Aim', alongside improving the health of populations, and reducing per capita costs of healthcare. ${ }^{3}$ Implementing a patient-centred approach is therefore necessary towards improving and achieving a high level of healthcare quality.

Although patient-centredness is changing the way healthcare systems around the world operate, healthcare performance measurement has yet to truly incorporate this patient-centred dimension of healthcare quality. The importance of systematic measurement and feedback to achieving patient-centred care is useful for monitoring and guiding improvement within organisations, as well as for holding organisations accountable for their results through public reporting. ${ }^{4}$ Healthcare quality indicators provide information that can be used to assess the quality of care, and are essential to optimise healthcare quality, track improvements and establish quality of care benchmarks, leading to changes in the care provided. ${ }^{5}$ The WHO has recently created the WHO global strategy on people-centred and integrated health services, ${ }^{6}$ but has noted: 'as of yet there are no universally accepted indicators to measure progress in establishing integrated people-centred health services'.

For the most part, quality has been measured in terms of service and system performance without incorporating the patient perspective, needs or values. Healthcare quality indicators from and/or incorporating patient perspectives are lacking and have not been routinely integrated into the evaluation of healthcare system performance; rather, system performance measurement has traditionally relied on routinely collected administrative and clinical data to monitor procedures, drug and treatment interventions, and outcomes. ${ }^{7-9}$ It has also been argued that patients lack the expertise to evaluate care received. ${ }^{10}{ }^{11}$ However, positive patient experiences have been linked to improved patient health status, while negative patient experiences have been shown to adversely affect health outcomes. ${ }^{12-14}$

The overall understanding of patient-centred care also varies from patients to providers to decision-makers. For instance, patients may value communication with healthcare providers, whereas providers may be more concerned about the effectiveness of treatment, and decision-makers may tend to focus on issues that affect the system, such as readmission rates and resource use. Thus, it is important to identify and implement measures that are relevant to patients and reflect their needs and values. It is also important to include the perspectives of family members and/or caregivers, who provide support to patients and may help to advocate on their behalf. In summary, in order to guide healthcare policy and implement practice change, healthcare systems need to develop and implement cost-effective and efficient ongoing mechanisms to measure and evaluate healthcare quality that incorporates all of these perspectives.

\section{STUDY RATIONALE}

Globally, healthcare policy and programmes have begun to promote patient-centred care models that could be implemented, measured and improved. The importance of measuring the effectiveness of patient-centred care has been identified as a need by multiple organisations ${ }^{1-3} 6$ in the process of transitioning towards the implementation of a patient-centred care healthcare model. However, despite a large number of heterogeneous, disease and setting-specific indicators that have been proposed, it is unclear the extent to which these indicators have been tested, implemented or validated. Further, there is currently no single set of generic indicators that exist which can be implemented to measure patient-centred care across multiple disease groups or care settings. There is no existing published synthesis on patient-centred quality indicators (PC-QIs) that incorporates both the peerreviewed and non-peer-reviewed literature.

\section{STUDY OBJECTIVES}

The objectives of this scoping review are to systematically scope the literature on PC-QIs, identify specific characteristics of these indicators and their definitions, and map indicators according to the thematic domains by which they can be classified. The indicators will be classified according to a predefined conceptual quality improvement framework adapted by the study team. Through this process, we will generate a comprehensive and well-defined list of unique indicators that may inform the adoption of standardised patient-centred care indicators. This work will constitute the first step in a multistep research programme aimed at the development of evidence-informed quality indicators to measure and evaluate the implementation and practice of patient-centred care across the continuum of care; the indicators determined through this review will subsequently be validated through a consensus review process. This information will allow healthcare organisations to assess the quality of patient-centred care being delivered, and provide outcome measurement data that can be used by healthcare system regulators and healthcare professionals to target efforts to improve care and service delivery that reflects what patients need and want.

\section{METHODS AND ANALYSIS}

\section{Conceptual model}

Our scoping review will use the Donabedian conceptual framework ${ }^{15}$ for assessing the quality of care using structure, process and outcome components of quality, to map PC-QIs and categorise them according to relevant thematic domains. This framework will serve as a guide for synthesising the literature and determining how quality indicators can be classified. Structure in this context encompasses the physical setting and organisational characteristics in which healthcare is provided, including materials and health resources; process comprises the methods by which healthcare is provided, and is 
dependent on the structures to provide resources and mechanisms to carry out care, therefore directly resulting in and impacting patient outcomes; outcomes are the result of healthcare provided and include the health status and experiences of patients and communities. Although Donabedian's model does not take into account specific patient factors, ${ }^{16}$ we have selected it because this model is perhaps the most widely used 'gold' standard for guiding quality improvement activities in healthcare. Specifically, this model has been used to outline the potential mechanisms of variation in quality and applied across a spectrum of healthcare settings and disease diagnoses, as well as being used to operationalise other types of healthcare quality measures (ie, Agency for Healthcare Research and QualityQuality Indicators). ${ }^{17}$

A second dimension of indicator classification that will be applied, in conjunction with the Donabedian model, will include patient-centred care-relevant domains taken from existing frameworks and evidence of domains in patient-centred care, for instance (but not limited to) the Picker Institute's Eight Principles for Patient-Centred Care $^{18}$ and the British Columbia Patient-Centered Care Framework. ${ }^{19}$ To illustrate how we will apply Donabedian's model and patient-centric domains, we have provided an example in table 1 . For example, patient-provider communication is an important thematic domain for patient-centred care; for the Donabedian component structure, an indicator example would be the development of an electronic-health or 'e-health' information technology system to support the communication between patients and healthcare providers across the continuum of care.

\section{Protocol design}

Methods for this study were developed based on Arksey and O'Malley's ${ }^{20}$ scoping review methodology, and Levac et $a l \mathrm{~s}^{21}$ methodological enhancement. According to this framework, there are six different stages in undertaking a scoping review: (1) identifying the research question; (2) identifying relevant studies; (3) selecting studies; (4) charting the data; (5) collating, summarising and reporting the results and (6) consulting with relevant stakeholders.

\section{Stage 1: Identifying the research question}

Through consultation with the research team and key stakeholders, the overall main research question developed is defined as: 'What are the quality indicators that have been implemented and evaluated across various points-of-care settings, processes of care and systems level proposed or currently in use to measure patient-centred care?'. For the purpose of this review, a quality indicator is 'an explicitly and measurable item which act as building blocks in the assessment of care'. ${ }^{22}$

\section{Stage 2: Identifying relevant studies}

\section{Search strategy and information sources}

Identification of studies relevant to this review will be achieved by searching electronic databases of the published literature which will include: MEDLINE, EMBASE, the Cochrane Library, Cumulative Index to Nursing and Allied Health Literature (CINAHL), PsycINFO, Social Work Abstracts and Social Services Abstracts. (The proposed search strategy is shown in online supplementary appendix 1'.) We will also handsearch all reference lists of included studies to identify additional studies of relevance.

To ensure that all relevant information is captured, we will also search a variety of grey literature sources. We will search relevant grey literature databases (eg, Grey Literature Report, OpenGrey, Web of Science Conference Proceedings) to identify studies, reports and conference abstracts of relevance to this review. We will also conduct a targeted search of the grey literature in local, provincial, national and international organisations' websites and related health or scientific organisations including: Patients Canada ${ }^{23}$ the Patient-centered Outcomes Research Institute (PCORI) ${ }^{24}$

Search terms will be determined with input from the research team, research collaborators and knowledge users. The search strategy will be developed by an experienced research librarian and coauthor (DLL), and will be revised pending input from stakeholders. Specifically, our patient-partner will be consulted for contribution of specific search terms regarding patient-centred care for the aspects of measurement to search the grey literature, as we suspect there will be more relevant existing grey literature on this topic. To ensure that no bias occurs, the patient-partner will be blinded to the original search strategy developed by the research team. Database and other searches will combine terms from two themes: quality indicators (eg, quality indicators, QI, performance indicators) and patient-centred care (eg, patient centered, patient centred, patient centric, patient beliefs, culture, ethnicity). Terms will be searched as both

Table 1 Example of indicator classification using the Donabedian model and patient-centred care domains.

\begin{tabular}{|c|c|c|c|}
\hline \multirow{2}{*}{$\begin{array}{l}\text { Example of PCC } \\
\text { thematic domains }\end{array}$} & \multicolumn{3}{|l|}{ Donabedian framework of quality ${ }^{11}$} \\
\hline & Structure & Process & Outcome \\
\hline $\begin{array}{l}\text { 1. Patient-provider } \\
\text { communication }\end{array}$ & $\begin{array}{l}\text { Development of innovative e-health } \\
\text { information technology to support and } \\
\text { enhance patient-provider communication }\end{array}$ & $\begin{array}{l}\text { Listening and responding to } \\
\text { patients' concerns and } \\
\text { expectations of care }\end{array}$ & $\begin{array}{l}\text { Effective and timely } \\
\text { access to care and } \\
\text { consultation length }\end{array}$ \\
\hline
\end{tabular}


keywords in the title and/or abstract and subject headings (eg, MeSH, EMTREE) as appropriate. No language or date limits will be applied. Search results will be downloaded and imported into a custom-written Java software application called Synthesis, ${ }^{25}$ specifically created for systematic and scoping literature reviews.

\section{Stage 3: Study selection}

The review process will consist of two levels of screening: (1) a title and abstract review and (2) full-text review. For the first level of screening, two investigators will independently screen the title and abstract of all retrieved citations for inclusion against a set of minimum inclusion criteria. The criteria will be tested on a sample of abstracts prior to beginning the abstract review to ensure that they are robust enough to capture any articles that may relate to PC-QIs. Any articles that are deemed relevant by either or both of the reviewers will be included in the full-text review. In the second step, the two investigators will then each independently assess the full-text articles to determine if they meet the inclusion/exclusion criteria. To determine inter-rater agreement, Cohen's $\kappa$ statistic $^{26}$ will be calculated at both the title and abstract review stage and at the full article review stage. Any discordant full-text articles will be reviewed a second time and further disagreements about study eligibility at the full-text review stage will be resolved through discussion with a third investigator until full consensus is obtained.

Relevant studies will be included if they describe the concept of patient-centredness or patient-centred care, and describe quality measurement or indicators concerned with patient-centred care, which can be a single measure or a set of measures. These measures can include patientreported outcome and patient-reported experience measures. Studies included can be on any of (a) development; (b) implementation; (c) evaluation; or (d) comparative validation of such measures. Any type of study design (eg, randomised control trials, case-control study, prospective or retrospective cohort study, quasi-experimental, qualitative) will be included. Studies will be excluded if they describe or evaluate quality improvement indicators in healthcare that are not patient-centric.

\section{Stage 4: Data collection}

A data collection instrument will be developed by the research team to confirm study relevance and to extract study characteristics. Study characteristics to be extracted will include, but not be limited to: publication year, publication type (eg, original research), study design, country, patient population characteristics, healthcare setting, patient-centered definition, description of quality indicators including definition, numerator, dominator, psychometrics of the indicators (face validity, reliability, construct validity, risk adjustment), and whether patients were involved in the development of the indicators. This form will be reviewed by the research team and pretested by all reviewers before implementation to ensure that the form is capturing the information accurately. Data abstraction will be conducted in duplicate with two reviewers independently extracting data from all included studies. To ensure accurate data collection, each reviewer's independent abstracted data will be compared and any discrepancies will be further discussed to ensure consistency between the reviewers. The data will be compiled in a single literature review software program, Synthesis, and then downloaded into a single excel spreadsheet in Microsoft Excel software for validation and coding.

\section{Stage 5: Data summary and synthesis of results}

Since a scoping review can be used to map the concepts underpinning a research area and the main sources and types of evidence available, the aggregated findings provide an overview of the research rather than an assessment of the quality of individual studies. For our scoping review, the quality indicators identified and extracted will be coded based on the Donabedian model and thematic dimensions of patient-centred care as discussed above. From this, a list of candidate quality indicators for further development and potential systematic reviews will be presented.

\section{Stage 6: Consultation}

Levac et $a l^{21}$ suggest that the consultation stage provides opportunities for stakeholder involvement, providing insights beyond what is reported in the literature. To address the study's patient-centric approach, stakeholders, in particular a patient-partner, will be engaged throughout the study acting as a consultant and knowledge user. Specifically, she will provide input regarding the search strategy and grey literature search to integrate the patient voice in capturing what is important to a patient in addressing measurement. This targeted search will be informed by input from subject experts and our provincial, national and international collaborators in different jurisdictions at the national level with the Canadian Institute for Health Information, the provincial level with Health Quality Councils, and internationally consult with experts in patient-centred care and measurement in the UK, Australia and Sweden. In addition, our patient-partner will connect with patient networks to provide a summary of results.

\section{DISSEMINATION AND ETHICS}

This study will constitute the first step in a multistep research programme aimed at developing a set of evidence-based PC-QIs that can be used across the healthcare spectrum. The results from this scoping review will guide the next phase of a multifaceted research programme that will lead to development of a set of PC-QIs that can be implemented at the system level to measure and monitor patient-centred care.

Since the scoping review methodology consists of reviewing and collecting data from publicly available 
materials, this study does not require ethics approval. To facilitate knowledge translation activities, our research team will use an integrated approach involving consulting a patient-partner to guide the research objectives and presenting the findings to other key health system stakeholders.

Contributors MJS conceived of the idea, developed the research question and study methods and contributed meaningfully to the drafting and editing; she has also approved the final manuscript. RJJ, DLL, KM, ML and HQ aided in developing the research question and study methods, contributed meaningfully to the drafting and editing, and approved the final manuscript.

Funding This work is being supported by an MSI (Medical Services Incorporated) Foundation grant (Canada).

Competing interests None declared.

Provenance and peer review Not commissioned; externally peer reviewed.

Data sharing statement No additional data are available.

Open Access This is an Open Access article distributed in accordance with the Creative Commons Attribution Non Commercial (CC BY-NC 4.0) license, which permits others to distribute, remix, adapt, build upon this work noncommercially, and license their derivative works on different terms, provided the original work is properly cited and the use is non-commercial. See: http:// creativecommons.org/licenses/by-nc/4.0/

\section{REFERENCES}

1. Shaller D. Patient-centred care: what does it take? The Commonwealth Fund, 2007

2. Institute of Medicine. Crossing the Quality Chasm: A New Health System for the 21st Century. Washington, DC: National Academy Press; 2001.

3. Berwick DM, Nolan TW, Whittington J. The triple aim: care, health, and cost. Health Aff (Millwood) 2008;27:759-69.

4. Hafner JM, Williams SC, Koss RG, et al. The perceived impact of public reporting hospital performance data: interviews with hospital staff. Int J Qual Health Care 2011;23:697-704.

5. Lawrence M, Olesen F. Indicators of quality health care. Eur J Gen Pract 1997:3:103-8.

6. World Health Organization. WHO global strategy on people-centred and integrated health services. Interim report. 2015 (accessed May 2016). http://apps.who.int/iris/bitstream/10665/155002/1/WHO_HIS SDS_2015.6_eng.pdf?ua $=1 \& u a=1$

7. Burge Fl, Bower K, Putnam W, et al. Quality indicators for cardiovascular primary care. Can J Cardiol 2007;23:383-8.
8. Santana MJ, Stelfox HT. Quality indicators used by trauma centers for performance measurement. J Trauma Acute Care Surg 2012;72:1298-302.

9. AHRQ Guide to Inpatient Quality Indicators: Quality of Care in Hospitals-Volume, Mortality, and Utilization. http://www. qualityindicators.ahrq.gov/downloades/modules/iqi/v31/iqi/iqi_guide_ v31.pdf (accessed 3 Jun 2016)

10. Nelson EC, Hays RD, Larson C, et al. The patient judgment system: reliability and validity. Qual Rev Bull 1989;15:185-91.

11. Patwardhan $\mathrm{A}$, Spencer $\mathrm{CH}$. Are patient surveys valuable as a service improvement tool in health services? An overview. J Healthc Leadersh 2012;4:33-46.

12. Fung $\mathrm{CH}$, Lim $\mathrm{YW}$, Mattke $\mathrm{S}$, et al. Systematic review: the evidence that publishing patient care performance data improves quality of care. Ann Intern Med 2008;148:111-23.

13. Kemp KA, Santana MJ, Southern DA, et al. Association of inpatient hospital experience with patient safety indicators: a cross-sectional, Canadian study. BMJ Open 2016:6:e011242.

14. Isaac T, Zaslavsky AM, Cleary PD, et al. The relationship between patients' perception of care and measures of hospital safety and quality. Health Serv Res 2010;45:1024-40.

15. Donabedian A. Explorations in quality assessment and monitoring. The definition of quality and approaches to its assessment. Vol 1. Ann Arbo, Ml: Health Administration Press, 1980.

16. McDonald KM, Sundaram V, Bravata DM, et al. Closing the quality gap: a critical analysis of quality improvement strategies (Vol. 7: Care Coordination). Rockville, MD: Agency for Healthcare Research and Quality (US), 2007. (Technical Reviews, No. 9.7.5), Conceptual Frameworks and Their Application to Evaluating Care Coordination Interventions. http://www.ncbi.nlm.nih.gov/books/NBK44008/ (accessed Jun 2016).

17. Agency for Healthcare Research and Quality AHRQ-Quality Indicators http://www.qualityindicators.ahrq.gov/ (accessed 1 Oct 2016).

18. Picker Institute's Principles of patient centred care. http://www. pickereurope.org/about-us/principles-of-patient-centred-care/ (accessed Jun 2016)

19. British Columbia Ministry of Health 2015. The British Columbia Patient-Centred Care Framework. http://www.health.gov.bc.ca/library/ publications/year/2015_a/pt-centred-care-framework.pdf (accessed Jun 2016)

20. Arksey H, O'Malley L. Scoping studies: towards a methodological framework. Int J Soc Res Methodol 2005;8:19-42.

21. Levac D, Colquhoun H, O'Brien KK. Scoping studies: advancing the methodology. Implement Sci 2010;5:69.

22. Campbell SM, Braspenning J, Hutchinson A, et al. Research methods used in developing and applying quality indicators in primary care. Qual Saf Health Care 2002;11:358-64.

23. Patients Canada. http://www.patientscanada.ca/ (accessed Jun 2016)

24. The Patient-centered Outcomes Research Institute. http://www.pcori. org/ (accessed Jun 2016).

25. Synthesis. http://synthesis.info/. Updated 2016.

26. Cohen J. A coefficient of agreement for nominal scales. Educ Psychol Meas 1960;20:37-46. 